\title{
Toward a National Standard in Primary Mass Casualty Triage
}

\author{
John H. Armstrong, MD, FACS, Erik R. Frykberg, MD, FACS, and David G. Burris, MD, FACS
}

Out of intense complexities, intense simplicities emerge

\section{—Winston Churchill}

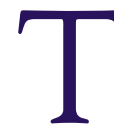
riage, or "to sort," had its genesis in war. In battles marked by frontal assault tactics, fields were covered with the wounded and the dead during and after the battle. Until the Napoleonic wars, however, there was no triage: medical care was not routinely offered on the battlefield, and the survivors self-identified through the test of time. Separating the living from the dead then occurred in the days after the battle ended. ${ }^{1}$

Baron Dominique Jean Larrey, Napoleon's chief surgeon, changed the approach to the wounded by practicing triage, treatment, and evacuation on the battlefield during combat. His guiding triage principle was that "It is necessary to always begin with the most dangerously injured." 2 Using his "flying ambulance," he saw medical evacuation as "the salvation of the injured and the conservation of the morale of the soldier."2

Field triage evolved over the conflicts of the next century from the prioritization of the most severely injured to a focus on achieving the greatest good for the greatest number as a way to conserve fighting strength. The wounded were thus categorized in a way to separate the living and salvageable from the dead and unsalvageable, within the overarching circumstances of resource availability. In the 20th century, advances included the development of rapid aeromedical evacuation to full-service field hospitals through the conflicts in Korea and Vietnam. ${ }^{3}$ In the current global war on terrorism, the scope and scale of conflict have changed from producing historical intermittent mass casualties in the thousands to daily "limited mass casualties" in the tens to low hundreds.

Wartime lessons contributed domestically to the creation of trauma centers, use of aeromedical transport from the injury scenes, and the development of standardized trauma evaluation and resuscitation, such as the Advanced Trauma Life Support program. With plentiful resources in the homeland, field triage was reframed to match individual patient injuries to trauma system resources to gain the best outcome for each individual patient. Such an approach is highlighted in an updated field triage decision scheme from the American
College of Surgeons Committee on Trauma in 4 steps across physiology, anatomy, mechanism, and special considerations, with the goal to determine which patient needs a trauma or other specialty center. ${ }^{4}$

In a mass casualty event, the temptation exists to do what we do everyday, just more of it. We tend to focus on the familiar individual casualty characteristics without appreciating that the application of triage in mass casualties varies by casualty load and resource availability. When we use the word "triage" to describe a decision-making process, we forget the different frames of reference (individual patient or mass casualties) and variable contexts (full or scarce resources) that must be specified (Fig. 1). This misunderstanding is then translated into mass casualty triage instruments: Although mass casualty triage is different from the daily triage of individual trauma patients, triage instruments retain a focus on casualties as individual patients.

The goal in the management of a mass casualty event is to achieve the greatest good for the greatest number of casualties. Nonbiological mechanisms of mass casualty produce the polar opposite outcomes of death or "the walking wounded," leaving up to $20 \%$ of living casualties critically injured and requiring immediate attention. These casualties are different from the usual trauma patients: they have multiple injury mechanisms producing complex, multidimensional injuries. The outcome that best reflects the medical care system in a mass casualty event is the critical mortality rate, or the percentage of deaths among the salvageable critically injured. ${ }^{5}$ These critically injured individuals have an injury severity score $>15$ as an index of injury severity that correlates with mortality. Triage is a population-based systemic process that integrates care in phases across sequential settings (scene, prehospital, hospital, region facilities) ${ }^{6}$ and must be designed to identify people who are critically injured amidst the sea of the walking wound. Toward this end, each setting has a twist on the triage process.

Five adult and 2 pediatric primary triage instruments have been developed for field management of civilian mass casualties. All of the instruments assess physiology and mentation at the scene, although the specific means for these assessments vary. These instruments have proliferated based on preference and politics, and have led to a patchwork of mass 


\section{FIGURE}

\section{Spectrum of triage scenarios as the situation moves from the individual patient to the casualty population and resources vary from plentiful to scarce.}

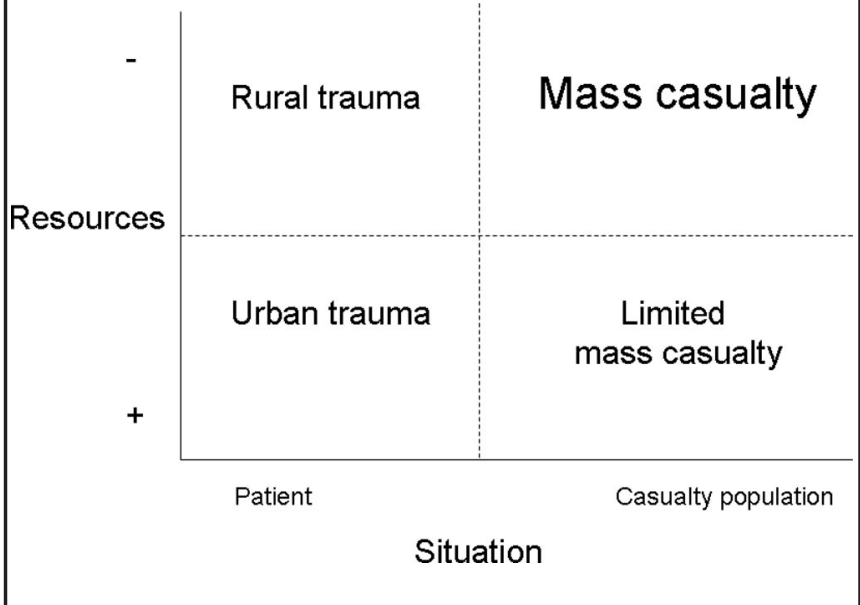

casualty triage systems in the United States and around the globe. ${ }^{7,8} \mathrm{~A}$ consistent lesson from the world of disasters is that the lack of an interoperable and consistent system of communication produces poor outcomes. Triage, as a communication system, falls under this lesson.

What are the key performance characteristics by which triage instruments should be examined to determine a standard guideline for triage?

- Simplicity, for execution in chaos

- Time efficiency, when time equals lives

- Predictive validity, so that the assessment relates to the intended outcome, namely, the identification of the critically injured from the mass of walking wounded

- Reliability, in that it is reproducible (with both the same rater and between raters) across all hazards

- Accuracy, to minimize over- and undertriage

Looking at the existing "mass casualty triage" instruments, and adding US military triage, the MASS triage training paradigm from the National Disaster Life Support program, and the French Red and White global response plans to the list, no instrument or plan has a strong evidence base, and studies of effectiveness in mass casualty situations are absent. So how do we achieve a common language in triage, a national standard for clinical and public health preparedness, in the existing potpourri of triage instruments that exist across jurisdictions and agencies?

This is the starting point for the work, in this issue, of Lerner and colleagues, who formed a consensus committee of representatives from the public and private sectors in emergency medicine, surgery, public health, and emergency medical services and worked to find an existing solution from the available triage instruments for primary field triage in mass casualty situations. ${ }^{9}$ The committee confirmed that validation efforts of existing triage instruments used paper exercises related to training; "retrospective triage" of individual, sequential trauma patients who were then grouped for analysis; simulation with outcomes defined by expert consensus; or prospective triage assessments of individual trauma patients over months in an emergency room. The instruments themselves missed the scale of the mass casualty, used an approach of serial rather than parallel casualty assessment, and mistook efficacy for effectiveness by using data collection in settings and situations that do not compare to mass casualty events and expert opinion to define reference standards. Collectively, the evidence for existing triage instruments in mass casualty events was no better than level V (expert opinion). ${ }^{10}$

Not finding an off-the-shelf solution, the committee designed a new instrument by incorporating elements from existing instruments to find a common denominator. Enter SALT: sort, assess, lifesaving interventions, treatment and/or transport. Picture a field with hundreds of casualties, akin to the traditional battlefield. What does SALT do that other triage instruments do not?

- Focuses first on the mass of casualties by voice command sorting. Hearing loss and self-transport of the walking wounded to the nearest hospital ${ }^{11}$ may limit controlled casualty distribution from the scene.

- Assesses casualties briefly for explicitly defined lifesaving interventions with applicability in chemical and radiation hazards. Controlling hemorrhage, opening airway, decompressing the chest (for tension pneumothorax), and autoinjection for chemical injury are actions triggered by brief sensory observations.

- Separates expectant from dead with a new color, gray. SALT emphasizes the relative nature of the expectant category based on available resources and the need for comfort care. Those casualties who are absolutely unsalvageable are unlikely to move out of the expectant category.

- Includes all ages; this instrument applies to adults and children, adding simplicity.

SALT seems to be simple to remember. Such clarity should facilitate standardized interdisciplinary training to competency in primary triage. ${ }^{12}$ Yet this proposal has the same limitations as the other triage instruments from which it is derived: it is level $\mathrm{V}$ evidence that needs study, through full-scale exercises and computer modeling, for validation and assessment of reliability, accuracy, and effectiveness. How will it stand up to reality?

One reality that bears emphasis is that scenes are inherently dangerous. Thus, the first priority of responders must be to move the living away from the scene to safety. Lifesaving interventions precede further classification through primary triage, which would then occur at the casualty collection point adjacent to the scene. Triage is not treatment, and the 
L-T part of SALT must only include those treatments that do not absorb time away from other casualties. Prehospital triage must initially sort casualties into 2 groups, the living and the dead, and then sort the living into those who need hospital care (and transport) and those who do not. The efficiency of SALT (or any triage instrument) to reorganize the scene is important for outcome, given the Israeli experience that 5 minutes of chaos requires 30 minutes to restore order. ${ }^{13}$

It is also important to put any primary triage instrument into the context of the triage system. Certainly, the primary triage decision sets conditions for success downstream in the response. Yet without a defined care pathway of sequential re-triage (secondary, tertiary, and beyond) of casualties, initial triage errors will fester and worsen population outcomes. The primary triage instrument does not work in a vacuum and must be linked in the triage system to the follow-on instruments. Furthermore, fractionating outcome to a particular triage instrument misses the point regarding the need for an error-tolerant triage system ${ }^{5}$ to overcome human error, changing casualty status, and resource fluctuations. Maintaining a consistent tempo through triage decisions-identifying who needs higher levels of care and who does notsupports a coherent triage system.

Gaps in triage knowledge persist. Benchmarks for triage accuracy do not exist. Communication across care settings is flawed-adequate documentation "provides the developing story to the next caregivers in line,"14 yet triage is too often seen as colored tags that are inviolate and indestructable. Confusion and discomfort persist regarding what "expectant" means, and guidelines for identifying absolute nonsalvageability are missing. Who should perform mass casualty triage across settings and how these multidisciplinary professionals should be trained as triage officers remain ripe for investigation.

There is no pretense among the authors that SALT is perfect or novel. This work adds urgency to do something to reach a national consensus on a unified approach to primary triage. SALT is a reasonable place to begin and overcomes the stasis preserved by latent parochialism. We should remember that sometimes the enemy of good is better. Perhaps we will then see the simplicity of getting to "yes" in consensus as a better way to prepare for the complexity of mass casualties.

\section{About the Authors}

Dr Armstrong is Assistant Professor of Surgery and Dr Frykberg is Chief of the Division of General Surgery, University of Florida; Dr Burris is Chairman of the Norman M. Rich Department of Surgery, Uniformed Services University of the Health Sciences.

Received for publication July 1, 2008; accepted July 2, 2008.

\section{Authors' Disclosures}

The authors report no conflicts of interest.

ISSN: 1935-7893 (C) 2008 by the American Medical Association and Lippincott Williams \& Wilkins.

DOI: 10.1097/DMP.0b013e31818582a5

\section{REFERENCES}

1. Iserson K, Moskop J. Triage in medicine: I. Concept, history, and types. Ann Emerg Med. 2007;49:275-281.

2. Burris D, Welling D, Rich N. Dominique Jean Larrey and the principles of humanity in warfare. JACS. 2004;198:831-835.

3. Mitchell G. A brief history of triage. D isaster Med Public Health Preparedness. 2008:2(Suppl 1):SX-SX.

4. Committee on Trauma, American College of Surgeons. Prehospital trauma care. In: Resources for Optimal Care of the Injured Patient 2006. Chicago: American College of Surgeons; 2006:21-25.

5. Frykberg E. Triage: principles and practice. Scand J Surg. 2005;94:272278.

6. Bostic N, Subbarao I, Burkle F, Hsu E, Armstrong J, James J. Disaster triage systems for large-scale catastrophic events. Disaster Med Public Health Preparedness. 2008:2(Suppl 1):SX-SX.

7. Jenkins J, McCarthy M, Sauer L, et al. Mass-casualty triage: time for an evidence-based approach. Prehosp Disaster Med. 2008;23:3-8.

8. Cone D, Koenig K. Mass casualty triage in the chemical, biological, radiological, or nuclear environment. Eur J Emerg Med. 2005;12:287302.

9. Lerner E, Schwartz R, Coule P, et al. Mass casualty triage: an evaluation of the data and development of a proposed national guideline Disaster Med Public Health Preparedness. 2008:2(Suppl. 1):SX-SX.

10. Wright J, Swiontkowski M, Heckman J. Introducing levels of evidence to the journal. J Bone Joint Surg Am. 2003;85:1-3.

11. Auf der Heide E. The importance of evidence-based disaster planning. Ann Emerg Med. 2006;47:34-49.

12. Subbarao I, Lyznicki J, Hsu E, et al. A consensus-based educational framework and competency set for the discipline of disaster medicine and public health preparedness, Disaster Med Public Health Preparedness. 2008;2:57-68.

13. Stein M, Hirshberg A. Medical consequences of terrorism: the conventional weapon threat. Surg Clin North Am. 1999;79:1537-1552.

14. Armstrong J, Burris D. Triage. In: Asensio J, Trunkey D, Current Therapy of Trauma and Surgical Critical Care. Philadelphia: Mosby Elsevier; 2008:55-57. 